\title{
A CLASSIFICATION FRAMEWORK FOR DRUG RELAPSE PREDICTION
}

\author{
A. K. M. Salleh ${ }^{1}$, M. Makhtar ${ }^{1, *}$, J. A. Jusoh ${ }^{1}$, P. L. Lua ${ }^{2}$ and A. M. Mohamad ${ }^{3}$ \\ ${ }^{1}$ Faculty of Informatics and Computing, Universiti Sultan Zainal Abidin, 21300 Gong Badak, \\ Terengganu, Malaysia \\ ${ }^{2}$ Faculty of Health Sciences, Universiti Sultan Zainal Abidin, Universiti Sultan Zainal Abidin, \\ 21300 Gong Badak, Terengganu, Malaysia \\ ${ }^{3}$ Faculty of General Studies and Continuing Education, Universiti Sultan Zainal Abidin, \\ 21300 Gong Badak, Terengganu, Malaysia
}

Published online: 10 November 2017

\begin{abstract}
This paper proposes a framework for relapse prediction using Artificial Neural Network algorithms among drug addicts at Pusat Rawatan Inabah. The data collected will be mining through Artificial Neural Network algorithms to generate patterns and useful knowledge and then automatically classifying the relapse possibility. This research collaborates with Pusat Rawatan Inabah, which is one of the rehabilitation centers that provide a specific treatment to rehabilitate the drug addicts from addiction. We expect that among the classification data mining algorithms, Artificial Intelligence Neural Network (ANN) is one of the best algorithms to predict relapse among drug addicts. This may help the rehabilitation center to predict relapse individually and the prediction result is hoped to prevent drug addicts from relapse.
\end{abstract}

Keywords: classification; artificial neural network; drug addiction; Inabah rehabilitation.

Author Correspondence, e-mail: mokhairi@unisza.edu.my

doi: http://dx.doi.org/10.4314/jfas.v9i6s.55

\section{INTRODUCTION}

Relapse is a major clinical and research problem in the addiction field, yet has no solution on the operational. As reported, relapse makes a difference in duration and rates to the first 
relapse [1]. One of the characteristics of addiction is drug craving and relapse to abuse drug after a while of abstinent [2]. Drug abuse and relapse indicates learned associations among drug-associated discrete, contextual symptoms, drug taking and also drug pharmacological effects. Rehabilitating those drug addicts become more challenging and tricky due to relapse to drug abuse is hard to be avoided [3]. In drug addicts, drug seeking and relapse often starts from serious re-exposure to the drug, drug-associated cues and bad pressure [2]. Relapse actually can be predicted through an automated computer technology exist, data mining. Here, we discuss details about data mining and the algorithms commonly used.

Classification data mining is commonly used to group the data under certain category labelled as "Class". "Class" in classification data mining refers to the attribute or feature in a dataset, that plays the main role as the user would be interested most on. In short, users are able to simply identify the data under certain class. The "class" is always defined as dependent variable in statistics. In order to classify data from a dataset, classification algorithm creates a classification model that runs classification rules [4]. Classification techniques are the way to discover a new research category (Class) from a set of data where the research category is known. Meanwhile, cluster analysis techniques are the way to categorize the objects based on the similarity [5]. A few classifiers in classification will be discussed in detail as below.

\subsection{Artificial Neural Network}

Artificial Neural Network (ANN) also known as neural network is a statistical or computational model that runs the similar process as biological neural network in human brain [6]. ANN model is a replication of human brain model that is able to replicate the neuron's communication for data processing and learning from experiences (patterns). Furthermore, the key point of ANN is to do replication as human neuron by replacing it with the artificial neurons. The model will go through training phase; real data is used in order to make the model discover the relationship among those variables. This is a learning process for the model. During the training phase, the model is able to identify the variables mostly related to the output and then it affects the relationship based on priority [7]. ANN approaches applies same analogy as in biology learning and memory. The units' development in the model is the nodes, represented as a neuron and weighted connections just like the synapses in a human brain (biological system). Nodes are the simple information in the processing element. In ANN, the amount of nodes developed and the connection among them can fluctuate. The more neurons are developed, the more ability to memorize and somehow reduced the reasoning ability of the ANN. General principle of ANN is ANN should minimize the number of neurons that able to analyze the training data. The training phase allows to keep the 
learning process in each nodes connection [8]. Learning in neural network involves modifying the weights of the connection to minimize the cost function where cost function include an error term a measure on how close the connection's predictions are to the class labels. Additionally, neural networks have been reviewed as such a useful tool in decision making process in many medical applications. Always bear in mind that these tools can never replace human experts, but it does assist the experts in diagnosis process [9]. ANN contains computational nodes that do the same function as neuron in human brain. The nodes are connected to each other by link that contains adjustable weight. The weights are modified through the learning process during training data phase. This classifier is founded in early 20th century. Again, it is the best classification algorithm before the introduction of decision trees (will be discussed in detail later). As a result, ANN becomes the most widely used classification algorithm. However, despite all the advantages reviewed, there are a few disadvantages where this classifier needs much variables and its classification performance is very dependent and sensitive to the variables selected. The second major disadvantage is this classifier have a slow training and learning process and also the expensive computational. Thirdly, ANN has minimum model transparency because the model is developed from a virtual element, number of nodes and real-valued weights. So, it is difficult for the healthcare professional to understand [4].

\subsection{Naïve Bayesian Classifier}

Naïve Bayesian Classifier is one of famous classifiers in classification data mining. A complete algorithm that appoints a class of predefined set to an object under development based on the descriptive variables. The task is completed by using probability approach which calculates class probabilities and predicts the most probable classes [5]. This classifier is a probabilistic learning method based on Bayes' theory, where this classifier merges evidence from different data sources in order to compute the probability of the expectation. Here, if there are a few expectations to be considered, Bayes theorem plays the role by providing the probability of each expectation based on the dataset. In the training phase, a naïve Bayes classifier plays with the training data to estimate the probability distribution. Meanwhile, during the testing phase, this classifier uses this estimation value to calculate the post-probability from a given testing data. Then, each and every tested data is categorized using the expectation with the highest post-probability [10]. Naïve Bayesian classifier based on Bayes' theory is a probability statistical classifier. The term "naive" refers to the variables dependency among them with condition. One of the main advantages of using naïve Bayesian classifier is the speed of use. This is due to the simplicity of the algorithm among other 
classification algorithms which allows the ability to manage a large number of variables. Furthermore, this classifier only requires a small dataset to be trained during training phase. However, there is a major disadvantage of naïve Bayes where the attributes or variables in a dataset are independent to each other. This assumption is not applicable in many cases. The use of naïve Bayes as a classifier is able to increase the classification accuracy, although the variables independence assumption are break, it is used broadly in medical data mining [4].

\subsection{Decision Tree}

Decision Tree was introduced by Ross Quinland in 1979. It is also known as Iterative Dichotomiser (ID)3. C4.5 is one of the most widely used decision tree algorithms. Decision tree classifiers build a flowchart like tree shape, in a top-down, recursive, divide-and-conquer, manner. The main process in building a decision tree is the Attribute Selection Method (ASM). ASM search for variables whose sorting result is nearest to the original partitions by the class in the terms of class values. Selected variables will become the nodes in a decision tree. Building a decision tree is the training phase of classification. One of the main advantages of this classifier is the visualization of the data. The visual is easy to provide understanding of the whole structure of the data. Besides that, the major disadvantage is difficulties to build the decision tree when the data contain a huge number of variables. The tree built could be too complex to be understood. This problem can be resolved via tree prune approaches. This approaches is a useful technology even though there may be slightly minor error in the trees generated [4]. Decision tree has the capability of analyzing continuous dataset, but the data format must be transformed first to data in categorical format [9].

All 3 algorithms discussed above are the algorithms in data mining. Recently, data mining in predicting diseases in healthcare sector is becoming more important. This is due to the large volume of data in healthcare organizations from day to day which is impossible to be analyzed using traditional way. In order to provide better service and save cost at the same time, the healthcare organization should have the capability of analyzing numbers of data correctly and the result should be in real-time. In short, a medical dataset need to transform to knowledge (patterns). Here, data mining as one of the automated computer technology that is able to fulfil this loop. At the same time, the clinical data and other millions of data can be stored safely. Commonly, the diagnosis is done based on doctors' knowledge and experience rather than on the knowledge hidden in the dataset. However, the human diagnosis can lead to human errors and extra cost which will then affect the service provided to patients [9].

Among this 3 algorithms that discussed above, the study would like to implement ANN in the model to predict relapse among drug addicts. Since addiction and relapse to drug use are the 
activities under human brain control, the study choose ANN algorithms due to the replication of biological neural network in human brain. Hence, the prediction should be more precise and accurate. ANN will discover the patterns (knowledge) hidden in the drug addicts dataset before the relapse prediction can be done.

This study focuses on the patient who has received treatment from the rehabilitation center, Pusat Rawatan Inabah where the patient is previously a drug addict. The patient volunteered himself to come here. However, there are a few cases that the patient is sent by family. The patient came to this rehabilitation center to get rehabilitation and recovery from drug craving. In some cases, the family send the patient to the rehabilitation center due to the wild attitudes of the drug addicts. The patient might overreact because of drug craving. The fact that those who have completed the rehabilitation before, to repeat the drug abuse again is possible. These relapse cases are possible to happen because of the drug craving feeling and unsupportive environment [11]. The patient here will be rehabilitated using an Islamic approach, known as Inabah.

\subsection{Inabah Program}

The Inabah program is an exclusive program as an alternative way to resolve addiction problem using spiritual or religious-related approaches. This is a rehabilitation program for youngsters who focus on spiritual individually. The program activities are full of religious approaches such as prayer (compulsory), reciting Quran, zikr and others. Abah Anom had created rehabilitation approaches for drug addicts using Inabah term. The term is defined as 'returning to the right path (Allah SWT's path)'. The objective of this rehabilitation center is to assist the drug addicts to the right direction, religious path. In Malaysia, Inabah is carried out through repent shower, zikrullah, qiyam al-lail, fasting, prayers and also nice attitude based on Islamic guideline [12]. The religion has given a big impact in healthcare sector. In other word, religion can influence individual's thought. This idea triggers the setting of Inabah program. Rehabilitate drug abusers in the rehabilitation centers must avoid the drug craving feeling in order to eliminate the addiction. If this feeling can successfully be avoided, the drug abusers will normally be able to dismiss from the rehabilitation program [11].

With the increasing number of drug addicts from year to year, a question raises here is could drug addiction be cured or healed? According to the statistic published by Ministry of Home Affairs and the National Anti-Drug Agency (AADK), the number of drug addicts reported in year 2014 is 13,605 for new cases. The number has risen to 20,289 new cases for year 2015 [13]. Meanwhile, the relapse cases reported in the year 2014 are 8,172 cases and the number decreases for the following year 2015 with 6,379 relapse cases reported [14]. Drug addiction 
is able to be treated and can be successfully controlled. Furthermore, drug addiction can also be prevented by providing a healthy environment including spreading the effects of drug abuse and addiction on youngsters. Clearly stated that drug addiction can be healed, however the relapse cases still exist even the number of relapse cases reported has decreased. Perhaps, prediction can be made first to prevent the drug addicts from relapse. Here, one of the automated computer technologies can probably be developed to predict relapse cases. When the prediction can be done, the number of relapse cases may drop [15].

\subsection{Classification Data Mining Tools}

The data mining tool is a combination of fundamentals, theories, methods and algorithms. However, these software have one objective which to seek for patterns of knowledge by combining a set of algorithms for data analysis with automated tools that allow users to obtain the results and report visualized in an interface. Basically, there are 4 free and open source data mining tools that are the best in the market; KNIME, Orange, RapidMiner and WEKA $[16]$.

This research will be using WEKA as a tool to predict the patterns hidden from the dataset. In the following section will be discuss details about WEKA.

\subsection{Waikato Environment for Knowledge Analysis (WEKA)}

WEKA is an open source tool for data mining that was developed at University of Waikato, New Zealand. It is free software which can be accessed under GNU General Public License. Developed in Java, WEKA consists of a bunch of machine learning algorithms in order to overcome many data mining problems in real. The concept of WEKA software is developed from a set of visualization tools and algorithms for data analysis purpose. Furthermore, a number of packages built in the software are useful algorithms such as Filters, Classifiers, Clusters, Associations and Attribute Selection [17]. WEKA consists of a group of machine learning algorithms that able to be used with different processing environment which include classification and clustering [18]. As reported in [4], WEKA (version 3.4.15) consists of approximately 50 different classification algorithms and 5 different algorithms in clustering. WEKA has been used for prediction due to the high performance in discovering, predicting and data analysis [19].

Since WEKA is free software which means, it is easy to get the access and can be reached anytime. This research decided to implement the study model of data mining. Besides, WEKA is a widely-used software tools in data mining field. It has the capability to classify the patients (drug addicts) into 2 different classes; Relapse and Not Relapse depend on the variables and input in the dataset. 
However, there must be a valid reason why WEKA was chosen as a data mining tool in this research. As reported that the best result was achieved with WEKA and the Decision Trees techniques with $85.96 \%$ among 8 datasets [16].

According to [20], WEKA has a few interesting features:

i. Implement learning method to dataset and analyze its result to learn more about the dataset

ii. Learned models can be used to get predictions on new inputs

iii. Predictions can be selected based on the application of a few different learners and comparison on their performance.

\section{METHODOLOGY}

In order to develop a model that run, apply and implement a data mining algorithms, several processes must be considered as to gain the clean data and accurate results. The process includes data collection, data cleaning and transformation, data integration, and data analysis. These 4 processes are the fundamental process to produce a pattern (knowledge) through artificial neural network algorithms. Then, the relapse prediction is able to be done when the learning phase took place.

\subsection{Data Collection}

A patient's dataset was completed based on demographic data together with the status of the patients, either the patient is a relapse patient or a new patient. The dataset also consist of religious data, to capture the patterns of religiosity level. The data compiled will be used to compare the various classification algorithms in order to gain the highest accuracy. The aim is to predict the patients' tendency of drug craving, relapse case. The classification data mining algorithms used in this research is Artificial Neural Network that suit the best to the dataset.

The variables referring to the data of patients' who undergo the Inabah program in Pusat Rawatan Inabah. The study was limited to patients who are under Inabah rehabilitation at the rehabilitation center, Pusat Rawatan Inabah Sungai Ikan, Terengganu, Malaysia.

The study is using a secondary data collected by other researchers. The method of collecting data could be from monitoring a set of questionnaires and some of the interview questions. The questionnaire is distributed to the patients in Pusat Rawatan Inabah Sungai Ikan, Terengganu, Malaysia. The focus variables is either the patient is a new drug addict receiving treatment or had undergo an addiction treatment before. In other words, the study would like to know either the patient is a relapse case or new case. 
The participating patients are given a set of questionnaires and they need to answer them honestly, without haste and in a peaceful surrounding. The elements included in the questionnaires were:

- Religiosity

- Craving

- Withdrawal

- Hospital Anxiety and Depression Scale (HADS)

- Knowledge, Attitude and Belief (KAB)

- Sahlgrenska Academy Self-Reported Cognitive Impairment (SASCI)

- World Health Organization Quality of Life (WHOQOL-BREF)

From these elements, the data is collected and will be compiled carefully. This research starts with compiling the data as a preparation of data mining process. Those questions and answers are reviewed to ensure that the answers are reliable and relevant. This is an important step to avoid missing any data. The missing data will affect the prediction results.

\subsection{Data Cleaning and Transformation}

A compiled dataset will be reviewed to ensure that the data collected is clean and relevant. The raw data always in horror format (numbers, words, sentences) which require modification and enhancement for prediction matters. The data will be evaluated through data completeness. The missing data will be valued using statistical measure either parameter mean or median or decision tree. Hence, the expected values able to be predicted and the missing valued are filled. After that, the data from other researchers will be compiled and the physical screening will be done to eliminate the data redundancy and to check any value conflicts.

When the secondary data becomes the sample data, the whole data will be rearranged according to research requirement for prediction purpose. The dataset then will be exported to ARFF (Attribute-Relation File Format) format for the use of WEKA machine learning tools. WEKA is able to analyze the data in ARFF format. WEKA will classify the data using ANN algorithm to predict relapse possibility among the patients.

\subsection{Data Integration}

Data Integration is the process where different sources of data are combined and compiled together to become a single dataset, in ARFF format that compatible with the requirement of WEKA program input. Since the variables are huge, the critical part is to ensure that the data is combined, compiled, inserted missing value, correctly to get the accurate prediction. A sequence number is used as a unique number represent each and every single patient. So, the 
patients' data should be compiled correctly. Fig. 1 shows the integration of data between multiple researchers to create a single trusted dataset.

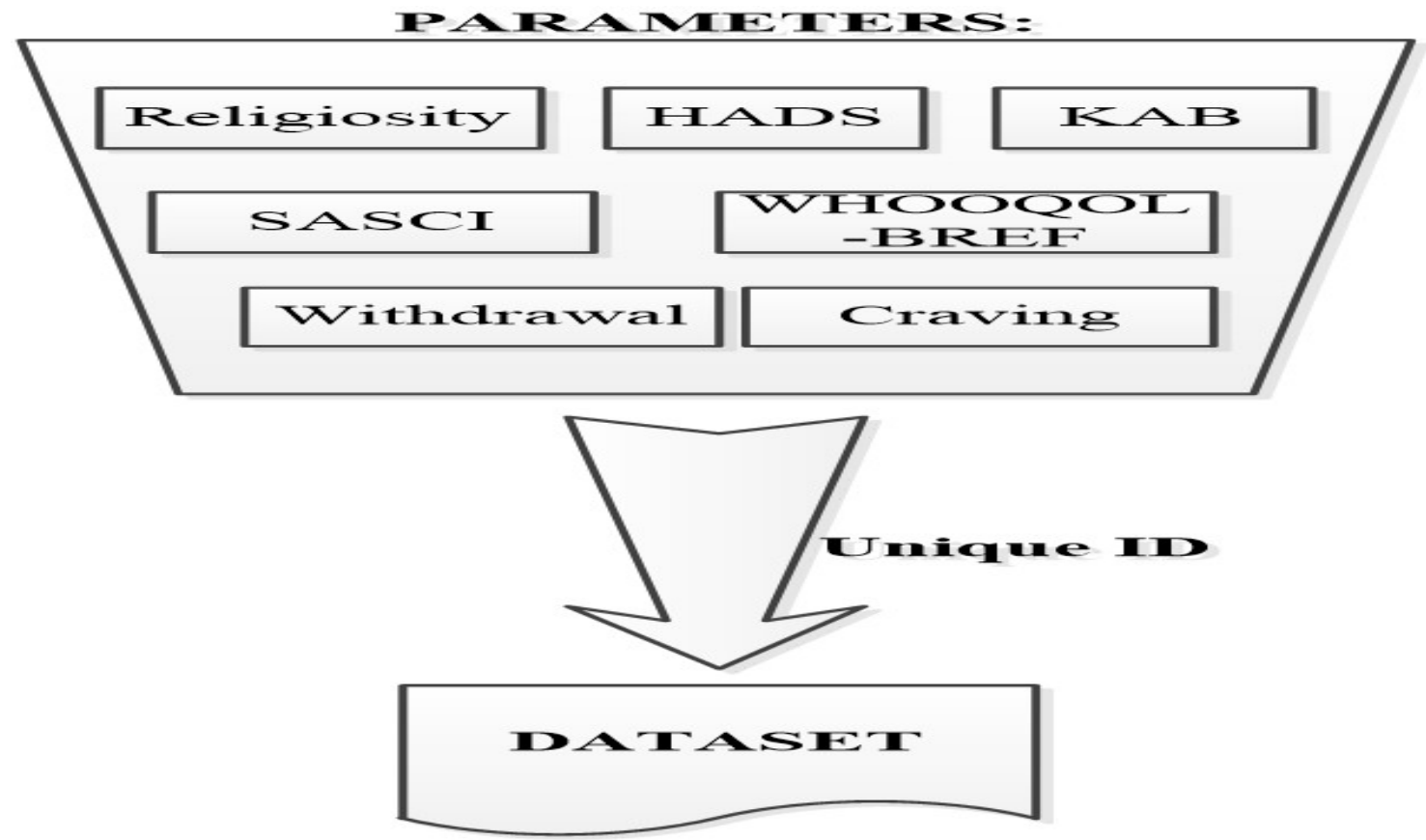

Fig.1.Data integration

As shown above, there are about 7 different data elements to be compiled in order to create a single dataset for relapse prediction purpose. The elements are Religiosity, Hospital Anxiety and Depression Scale (HADS), Knowledge, Attitude and Belief (KAB), Sahlgrenska Academy Self-Reported Cognitive Impairment (SASCI), World Health Organization Quality of Life (WHOQOL-BREF), Withdrawal and Craving. A brief description of each element will be described in Table 1. 
Table 1.Brief description of each data element

\begin{tabular}{ccl}
\hline No. & Data Element & \\
\hline 1. & Religiosity & $\begin{array}{l}\text { To measure the agreement with the beliefs/statements or frequency } \\
\text { of the performance }\end{array}$ \\
2. & HADS & To measure the anxiety and depression scale of each drug addicts \\
3. & KAB & To measure the level of knowledge and awareness and personal \\
& & familiarity with illegal drugs \\
4. & SASCI & To measure cognitive functioning among drug addicts \\
5. & WHOQOL-BREF & To measure quality of life among drug addicts \\
6. & Withdrawal & To determine the frequency of drug withdrawal symptoms. \\
7. & Craving & To determine intensity, frequency and duration of craving among \\
& & drug addicts
\end{tabular}

Each patient (instances) will be given a unique ID, a running number starting from 1 . The ID is given to avoid using their real name or IC number as an instances. This step is important to protect the patients' information (data integrity). Then, all the data of each element will be compiled referring the unique ID given to avoid wrong data compiled.

\subsection{Data Analysis}

Data analysis is the last step in the methodology. After a single clean and trusted data is produced, analysis will be done to the dataset in order to get the knowledge (patterns) hidden. This analysis can be done using one of classification algorithm tools, an automated tool, WEKA.

Artificial Neural Network (ANN) recently is one of the data mining algorithms that broadly-used in medicine and healthcare sector in predicting diseases. It has become one of the main algorithms in data mining research to seek for patterns of the variables that is the most influential in the research objective. Many researches had been successfully using ANN. The ANN was used to diagnose starbisme. The model is a web-based system that is available online (www.strabnet.com). Hence, the physician is able to directly submit required information to the system after examining the patients. Then, the diagnose part will be done by the system referring to the information received. The result produced shows that the accuracy of $100 \%$ on real data [21]. Multilayer neural network (with 19 input) with Levenberg Marquardt training algorithm was developed in order to do hepatitis prediction (live or die). The system accuracy of classification was $91.87 \%$ [22]. Meanwhile, the same data had been used which developed a probabilistic neural network. The accuracy was 91.2\% [23]. Regarding the successful of classification data mining and ANN research accuracy result in 
many researches, this study is hoped to discover the patterns hidden behind the drug addicts' dataset and at the same time to classify the possibility of relapse among drug addicts in Pusat Rawatan Inabah Sungai Ikan. As a result, the output of the research is hoped to help rehabilitation center to prevent the relapse case. Fig. 2 represents the conceptual framework for the whole research.

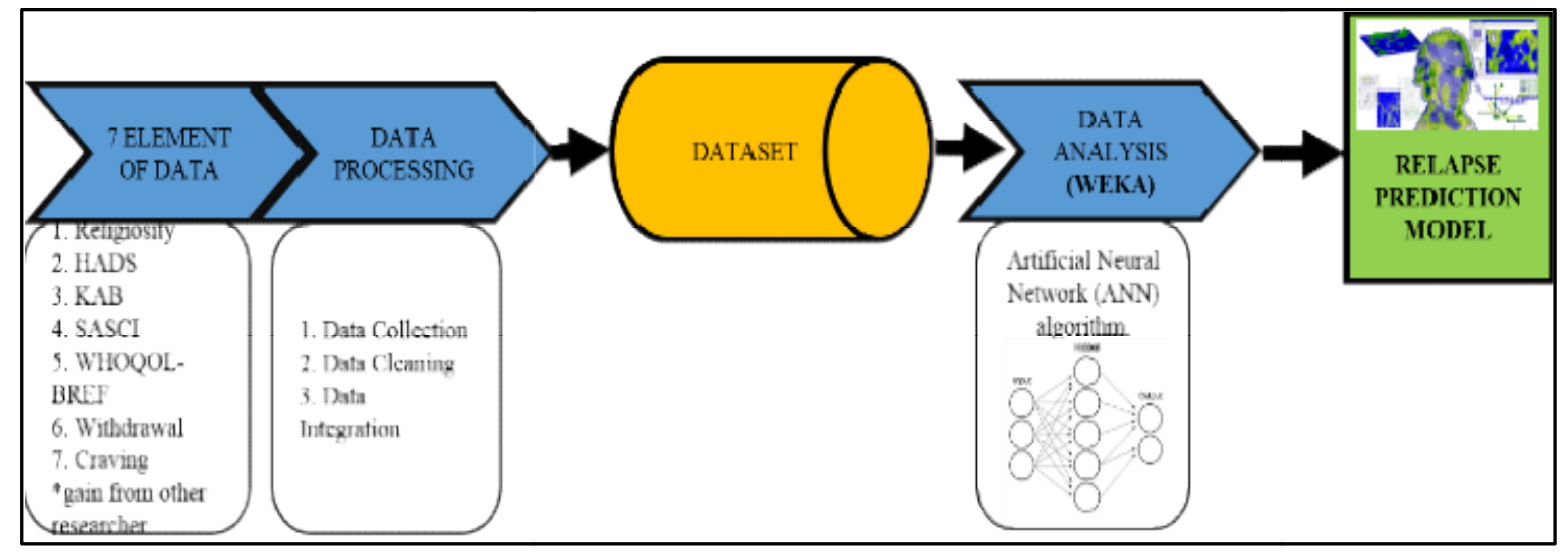

Fig.2.Conceptual framework for relapse prediction 
As described above, the research starts with gathering data from other researchers according to their field of research. The data is divided into 7 elements with different researchers on respective field of study. The elements are Religiosity, HAD, KAB, SASCI, WHOQOL-BREF, Withdrawal and Craving. All data (parameters) will be cleaned to get the raw data. To prepare a dataset for prediction purpose, data from 7 elements must be integrated into a single file to become as an input in data mining tools, WEKA. The analysis will be done to discover patterns (knowledge) hidden in the dataset using ANN algorithm. This phase will produce a number of parameters related to relapse. Then, a model will be developed to insert those parameters related to relapse. The model is expected to calculate the accuracy of relapse and display the prediction results of each drug addicts.

The objective of creating this conceptual framework is to provide basic guideline of the research and basic methodologies being used for this research. The framework has 3 major steps starting from receiving raw data from other researchers. Here, the research begins with the massaging the data to produce a set of data that ready for the next step. The massaging includes eliminating and modifying redundant, incomplete, duplicate and missing data. This process is important in order to ensure that the data is consistent and at the same time able to gain an accurate result. The ready dataset then will be analyzed using ANN algorithms embedded in WEKA tools to predict the relapse possibility.

\section{RESULTS AND DISCUSSION}

The data set is collected from other researchers. The collected data then will be compiled and the cleaning process takes place. All variables and parameters compiled are mandatory to be in numeric or nominal format before data mining start. By using an automated tool, WEKA, the data will undergo pre-process phase. WEKA provides a few classifiers to choose and the algorithm selected is Artificial Neural Network as it suits with the dataset as discussed before. Using classifier Artificial Neural Network, the data is expected to classify into 2 groups of data which are Relapse and Not Relapsegroup as stated in theresearch aim.Users are able to simply identify Relapse drug addicts and give them more attention in the rehabilitation process to avoid relapse from happening. The data produced is displayed through one of the computer technologies, friendly user interface. 


\section{CONCLUSION}

In this paper, we first reviewed a conceptual framework to predict relapse among drug addicts at Pusat Rawatan Inabah using Artificial Neural Network [24-27] algorithms. The research objectives are to apply data mining techniques with the help of intelligence technologies involving automated tools, WEKA.

Since day to day relapse cases reported, we would like to propose the automated tools to predict relapse among drug addicts. There are 8172 cases of relapse reported. The number decreased for the following year with only 6379 relapse cases reported [13]. It is issued by Agensi Anti-Dadah Kebangsaan Malaysia (AADK). It makes the decreasing percentage is $13.61 \%$ from whole case reported. This reducing percentage of relapse cases prove that drug addicts can be cured and the addiction can be avoided [14].

The data collected will go through a few processes before prediction can be made. The data is collected, clean-up, integrated and then analyzed. This research provides a foundation for data mining techniques that are able to help rehabilitation centers to prevent relapse from happening. The future work should consider a data set from drug addicts that undergo other than Inabah treatment.

\section{ACKNOWLEDGEMENTS}

This research is supported by UniSZA (Grant No. Unisza/1/2015/SRGS/1) and (Grant No. Unisza/1/2015/SRGS/2). The authors would like to thank Pusat Rawatan Inabah management for and all research team for sharing the data set.

\section{REFERENCES}

[1] Maisto S A, Pollock N K, Cornelius J R, Lynch K G, Martin C S. Alcohol relapse as a function of relapse definition in a clinical sample of adolescents. Addictive Behaviors, 2003, 28(3):449-459

[2] Marchant N J, Li X, Shaham Y. Recent developments in animal models of drug relapse. Current Opinion in Neurobiology, 2013, 23(4):675-683

[3] Bossert J M, Marchant N J, Calu D J, Shaham Y. The reinstatement model of drug relapse: Recent neurobiological findings, emerging research topics, and translational research. Psychopharmacology, 2013, 229(3):453-476 
[4] Yoo I, Alafaireet P, Marinov M, Pena-Hernandez K, Gopidi R, Chang J F, Hua L. Data mining in healthcare and biomedicine: A survey of the literature. Journal of Medical Systems, 2012, 36(4):2431-2448

[5] Thirumal P C, Nagarajan N. Utilization of data mining techniques for diagnosis of diabetes mellitus-a case study. ARPN Journal of Engineering and Applied Science, 2015, 10(1):8-13

[6] Sudhakar K, Manimekalai D M. Study of heart disease prediction using data mining. International Journal of Advanced Research in Computer Science and Software Engineering, 2014, 4(1):1157-1160

[7] Sheikhtaheri A, Sadoughi F, Dehaghi Z H. Developing and using expert systems and neural networks in medicine: A review on benefits and challenges. Journal of Medical Systems, 2014, 38(9):1-6

[8] Sinha S K, Wang M C. Artificial neural network prediction models for soil compaction and permeability. Geotechnical and Geological Engineering, 2008, 26(1):47-64

[9] Srinivas K, Rani B K, Govrdhan A. Applications of data mining techniques in healthcare and prediction of heart attacks. International Journal on Computer Science and Engineering, 2010, 2(2):250-255

[10]Spasić I, Burnap P, Greenwood M, Arribas-Ayllon M. A Naïve Bayes approach to classifying topics in suicide notes. Biomedical Informatics Insights, 2012, 5(Suppl 1):87-97

[11]Istiqomah I N. Reduced addiction in drugs abusers undergoing dhikr at Ponpes Inabah XIX Surabaya. Folia Medica Indonesiana, 2013, 49(1):8-11

[12]Puteh D A H M A, Ghani N A, Mamat I, Merbawi A M M A. Drug addict treatment and rehabilitation programme at Pondok Inabah, Kuala Terengganu, Terengganu, Malaysia. British Journal of Social Sciences, 2013, 1:37-46

[13]Home Affairs and the National Anti-Drug Agency (AADK). Maklumat dadah 2015. 2015, http://www.adk.gov.my/html/pdf/buku\%20maklumat\%20dadah/BUKU\%20MAKLUMAT\%2 0DADAH\%202015.pdf

[14]Home Affairs and the National Anti-Drug Agency (AADK). Maklumat dadah 2014. 2014, http://www.adk.gov.my/html/laporandadah/2014/FINAL\%20BUKU\%20MAKLUMAT\%20D ADAH\%202014.pdf

[15]National Institute on Drug Abuse. DrugFacts-Understanding drug use and addiction. 2016, https://www.drugabuse.gov/publications/drugfacts/understanding-drug-abuse-addiction 
[16]Borges L C, Marques V M, Bernardino J. Comparison of data mining techniques and tools for data classification. In ACM International C* Conference on Computer Science and Software Engineering, 2013, pp. 113-116

[17] Anuradha C, Velmurugan T. A comparative analysis on the evaluation of classification algorithms in the prediction of students performance. Indian Journal of Science and Technology, 2015, 8(15):1-12

[18]Barnaghi P M, Sahzabi V A, Bakar A A. A comparative study for various methods of classification. In International Conference on Information and Computer Networks, 2012, 27(2):875-81

[19] Masethe H D, Masethe M A. Prediction of heart disease using classification algorithms. In World Congress on Engineering and Computer Science, 2014, pp. 1-4

[20]Hasim N, Haris N A. A study of open-source data mining tools for forecasting. In ACM 9th International Conference on Ubiquitous Information Management and Communication, 2015, pp. 1-4

[21]Fisher A C, Chandna A, Cunningham I P. The differential diagnosis of vertical strabismus from prism cover test data using an artificially intelligent expert system. Medical and Biological Engineering and Computing, 2007, 45(7):689-693

[22]Bascil M S, Temurtas F. A study on hepatitis disease diagnosis using multilayer neural network with Levenberg Marquardt training algorithm. Journal of Medical Systems, 2011, 35(3):433-436

[23]Bascil M S, Oztekin H. A study on hepatitis disease diagnosis using probabilistic neural network. Journal of Medical Systems, 2012, 36(3):1603-1606

[24]Zabidi A, Yassin I M, Hassan H A, Ismail N, Hamzah M M, Rizman Z I, Abidin H Z. Detection of asphyxia in infants using deep learning convolutional neural network (CNN) trained on Mel frequency cepstrum coefficient (MFCC) features extracted from cry sounds. Journal of Fundamental and Applied Sciences, 2017, 9(3S):768-778

[25]Hashim F R, Daud N N, Ahmad K A, Adnan J, Rizman Z I. Prediction of rainfall based on weather parameter using artificial neural network. Journal of Fundamental and Applied Sciences, 2017, 9(3S):493-502

[26]Hashim F R, Adnan J, Ibrahim M M, Ishak M T, Din M F, Daud N G, Rizman Z I. Heart abnormality detection by using artificial neural network. Journal of Fundamental and Applied Sciences, 2017, 9(3S):1-10

[27]Yassin I M, Jailani R, Ali M, Baharom R, Hassan A, Rizman Z I. Comparison between cascade forward and multi-layer perceptron neural networks for NARX functional electrical 
stimulation (FES)-based muscle model. International Journal on Advanced Science, Engineering and Information Technology, 2017, 7(1):215-221

How to cite this article:

Salleh A K M, Makhtar M, Jusoh J A, Lua P L, Mohamad A M. A classification framework for drug relapse prediction. J. Fundam. Appl. Sci., 2017, 9(6S), 735-750. 